\title{
Enseñando Modelización a Nivel Universitario: la relatividad institucional de los recorridos de estudio e investigación
}

\author{
Teaching Modelling at University Level: \\ the institutional relativity of study and research paths
}

Berta Barquero*

\begin{abstract}
Resumen
Este trabajo se centra en extender el problema de la ecología de la modelización matemática en su enseñanza a nivel universitario. Nuestro principal objetivo es estudiar la variedad de restricciones que aparecen cuando actividades de modelización son llevadas a las aulas universitarias, impidiendo su evolución normal y estudiar las condiciones que permiten superar su rigidez. En el marco de la teoría antropológica de lo didáctico, la propuesta del diseño de los recorridos de estudio e investigación (REI) apunta nuevas posibilidades de superar algunas de estas restricciones. Presentamos, aquí, el diseño y experimentación de un REI basado en las dificultades de conseguir una distribución homogénea de bicicletas en un sistema urbano de uso compartido. Se presentarán las sucesivas transformaciones que sufre su diseño para adaptarse a dos entornos institucionales distintos y las respuestas y reacciones que estudiantes y profesores universitarios ponen de manifiesto ante dichas experimentaciones.
\end{abstract}

Palabras-clave: Modelización Matemática. Teoría Antropológica de lo Didáctico. Recorridos de Estudio e Investigación. Ecología. Relatividad Institucional.

\begin{abstract}
This paper focuses on extending the problem of the ecology of mathematical modelling activities at university level. Our aim is to deal with the variety of constraints appearing when modelling proposals, which are implemented in university classrooms impeding their normal lives and to study the appropriate conditions which can overcome them. Within the framework of the anthropological theory of the didactic, the proposal of the study and research paths (SRP) shows new possibilities to surmount some of these constraints. We present here the design and implementation of a SRP based on the difficulty to get a homogeneous distribution of bicycles in a bike-sharing system. This paper presents successive transformations that the design of a SRP' had to undergo to deal with two different institutional environments and the answers and reactions that university teachers and students expressed in front of its implementations.
\end{abstract}

Keywords: Mathematical Modelling. Anthropological Theory of the Didactics. Research and Study Paths. Ecology. Institutional Relativity.

\footnotetext{
* Doctora en Matemáticas por la Universidad Autónoma de Barcelona (UAB). Profesora e Investigadora en Didáctica de la Matemática. Departamento de Didáctica de las Ciencias Experimentales y la Matemática. Universidad de Barcelona (UB), Barcelona, España. Dirección postal: Paseo de la Vall d'Hebron, 171, HortaGuinardó, 08035, Barcelona, España. E-mail: bbarquero@ub.edu
} 


\section{Introducción: el problema de la ecología de la modelización matemática}

El punto de partida de este trabajo es el de seguir indagando en cómo y bajo qué condiciones es posible integrar actividades de modelización en los procesos de enseñanza y aprendizaje de las matemáticas $\mathrm{y}$, en particular, en la enseñanza universitaria de esta disciplina. En las últimas décadas, numerosas investigaciones han mostrado cómo las actividades de modelización matemática pueden desarrollarse bajo ciertas condiciones adecuadas en los distintos niveles educativos y en muchos contextos curriculares distintos. A pesar del importante progreso en este ámbito de investigación conocido como modelización y aplicaciones, la difusión y pervivencia de la modelización como una actividad central en los procesos de enseñanza y aprendizaje de las matemáticas, en todo nivel educativo, se mantiene como uno de los grandes retos de las últimas décadas para la Educación Matemática. Entre otros, Burkhardt (2006) enfatiza la existencia de dos universos: por un lado, el buen progreso y resultados prometedores en el campos de investigación y, por otro, las grandes dificultades y restricciones que nuestras aulas, en todo nivel educativo, tienen por integrar este tipo de actividades. Por su parte, Galbraith $(2007$, p.78) describe la situación usando una metáfora un tanto provocadora:

\footnotetext{
When the low hanging fruit is also sweet, the incentive to search the higher branches is diminished further. In the case of applications and modelling a shared excitement unites many who have enthused about early experiences in the field, for example when students unleash latent power that for whatever reason had remained fettered in their previous mathematical life. However, this very exhilaration can work against further progress, both individually, and particularly at a system level, by creating a sense of adequate achievement that obscures the reality that there is so much more to do.
}

El problema de investigación que nos propondremos en este trabajo requiere, en primer lugar, llevar a cabo un análisis epistemológico riguroso y preciso de qué se entiende por modelización matemática, para situarla dentro de un modelo epistemológico general de la actividad matemática. En el ámbito de investigación en el que nos situaremos, el de la Teoría Antropológica de lo Didáctico (TAD, en adelante), se asume que la modelización matemática debe formar parte integrante de cualquier proceso de estudio de las matemáticas puesto que esta actividad se considera como sinónimo de actividad matemática funcional en contraposición a la actividad matemática formal. Más concretamente, se propone describir los procesos de modelización como procesos de estudio, construcción y articulación de organizaciones matemáticas de complejidad creciente (puntuales, locales, regionales) con el objetivo de dar respuesta a ciertas cuestiones problemáticas que plantean en cierto ámbito de la realidad intra-matemática o extra-matemática (GARCÍA et al., 2006; BARQUERO; 
BOSCH; GASCÓN, 2011). Estas cuestiones problemáticas serán las que constituirán la razón de ser del proceso de estudio y la modelización matemática el motor e instrumento clave en el proceso de búsqueda, construcción y articulación de herramientas y modelos matemáticos, posibilitando a su vez la constante elaboración de respuestas cada vez más amplias y completas.

Asumiendo este concepto de modelización matemática propuesto des del ámbito de la $\mathrm{TAD}$, será crucial tomar en consideración la dimensión ecológica del problema de la modelización matemática, que significará asumir la existencia de condiciones y de restricciones que, con independencia de la voluntad de los sujetos, inciden sobre la vida institucional de las actividades de enseñanza y aprendizaje de la modelización matemática. Esta nos llevará a cuestionar y estudiar las condiciones de vida (génesis, desarrollo, relación, desaparición etc.) y de difusión de dichas prácticas en las distintas instituciones consideradas. Partiendo de las investigación previas citadas, el problema de investigación didáctica relativo a la enseñanza de la modelización matemática a nivel universitario puede formularse en los siguientes términos, incorporando de manera esencial las dimensiones epistemológica y ecológica inherentes (ARTAUD, 2007; GASCÓN, 2011):

\footnotetext{
¿Qué tipo de dispositivos didácticos posibilitarían una integración global (más allá de una experimentación local) de la modelización matemática en los actuales sistemas de enseñanza universitaria?

¿Cuál es la ecología de estas organizaciones didácticas? Esto es, ¿qué condiciones favorecen que la modelización matemática pueda existir y pervivir como una actividad normalizada en la enseñanza universitaria y qué restricciones impiden o dificultan que esto pueda ser posible?
}

Aparece, aquí, la propuesta de los recorridos de estudio e investigación (REI), introducidos por Chevallard $(2006,2013)$ cuyo diseño, implementación y análisis ecológico se situarán en la base de esta investigación. Se considera que un REI viene generado por el estudio de una cuestión inicial, que llamaremos cuestión generatriz $Q_{0}$, con fuerte poder generador capaz de plantear un gran número de cuestiones derivadas: $Q_{1}, Q_{2}, \ldots, Q_{n}$. El estudio de estas cuestiones va a conducir a la construcción de ciertas respuestas provisionales $R_{i}$ que delimitarán el mapa de los posibles trayectorias a recorrer y sus límites, aunque provisionales, en la elaboración de una respuesta final a $Q_{0}$. Se generará, así, una red de cuestiones y respuestas que se despliegan en una dialéctica compleja en la cual la construcción y estudio de modelos matemáticos jugará un papel fundamental en proporcionar las herramientas matemáticas necesarias para la elaboración de las sucesivas respuestas y su contraste con el medio experimental intra - o extra - matemático más adecuado. 


\section{La ecología de los REI y su relatividad institucional}

El problema de la ecología de la modelización matemática, en el caso especial de la propuesta de los REI para la enseñanza universitaria de las matemáticas, ha sido abordado en distintas investigaciones (WINSLØW et al., 2014). En particular, partimos de los trabajos a nivel universitario de Barquero, Bosch y Gascón $(2011,2013)$ en el caso de los estudios universitarios de Ciencias Experimentales (CCEE) y Serrano, Bosch y Gascón (2013) en el caso de Ciencias Económicas. Con este objetivo se han diseñado, implementado y analizado los REI como dispositivo didáctico para facilitar la inclusión de la modelización matemática y, más concretamente, para situar explícitamente los problemas de modelización matemática en el corazón de los procesos de enseñanza y aprendizaje de las matemáticas a nivel universitario. Dichas implementaciones y su posterior análisis han permitido, además de comprobar que el diseño de un REI permitía cubrir los contenidos de un curso anual de matemáticas, empezar a constatar la aparición de diversas restricciones cuando estos dispositivos se introducen en los sistemas de enseñanza universitarios. Entre otros, la necesidad de romper con la rigidez de la estructura clásica de teoría-sesión de problemasexamen y de articular el REI con los dispositivos tradicionales, la necesidad de nuevos dispositivos didácticos para dar cabida a un nuevo contrato didáctico y donde nuevas técnicas didácticas puedan tomar vida, la ausencia de un discurso matemático alternativo para referirse al trabajo de modelización (como las propias nociones de sistema, modelo, reformulación de las hipótesis y de los modelos etc.).

Por otro lado, en Barquero, Bosch y Gascón (2014) se muestra el grado de influencia e impacto que tiene la epistemología espontánea del profesorado de CCEE y de la pedagogía escolar dominante en las instituciones universitarias sobre las condiciones de vida de los REI y, por extensión, sobre la enseñanza de la modelización matemática en dichas instituciones. Esta influencia se materializa en un conjunto de restricciones que se derivan de la forma de interpretar la relación entre las matemáticas y las CCEE y del papel que se otorga a las matemáticas en la enseñanza de las CCEE. En el trabajo citado se caracteriza y contrasta hasta qué punto prevalece la epistemología dominante en la enseñanza universitaria de las matemáticas para las CCEE, que los autores denominan aplicacionismo, bajo la influencia del cual la noción de modelización se restringe enormemente para ser identificada como una mera aplicación del conocimiento matemático previamente enseñado.

En este trabajo nos proponemos dar un paso más para seguir profundizando en el problema de la ecología institucional de la modelización en la enseñanza universitaria de las 
matemáticas. Nos proponemos analizar distintos aspectos de la relatividad institucional de las organizaciones matemático-didácticas que puedan dar cabida a este tipo de actividades. Como se describe en Sierra (2006), y siguiendo los trabajos de Castela (2004) y Castela y Romo (2011), cada institución mantiene una relación institucional con los saberes matemáticos, por ello, cada institución impone un conjunto de restricciones y condiciones específicas en el proceso de estudio y construcción de los saberes, es decir, en la reconstrucción de las organizaciones matemáticas y didácticas correspondientes, en el sentido de la TAD.

Ciertos aspectos de esta relatividad institucional están estrechamente ligados a los modelos epistemológicos y pedagógicos dominantes de cada institución (SIERRA, 2006, p.111-121). Así, por ejemplo, si en una institución docente determinada, en nuestro caso la universitaria, se restringe enormemente la forma de interpretar la modelización matemática entendiéndola como una mera aplicación del conocimiento matemático previamente construido por el profesor o, en su caso más extremo, como una simple ejemplificación de las herramientas matemáticas en ciertos contextos extra-matemáticos artificialmente construidos ${ }^{1}$, entonces se estará dificultando que los estudiantes puedan desarrollar un trabajo autónomo donde propongan, construyan y desarrollen distintos modelos matemáticos, den sus propias respuestas provisionales a ciertas cuestiones problemáticas, exploren entre distintas posibilidades en base a su propio trabajo etc.

Será aquí donde pondremos nuestro foco principal. Nos proponemos empezar a analizar los efectos ligados a la relatividad institucional de los REI y, con ello, estudiar la emergencia, persistencia y alcance de las condiciones y restricciones que emergen en el proceso de diseño (o rediseño), implementación de los REI y análisis de sus puestas en práctica bajo variaciones institucionales. Más concretamente, nos planteamos:

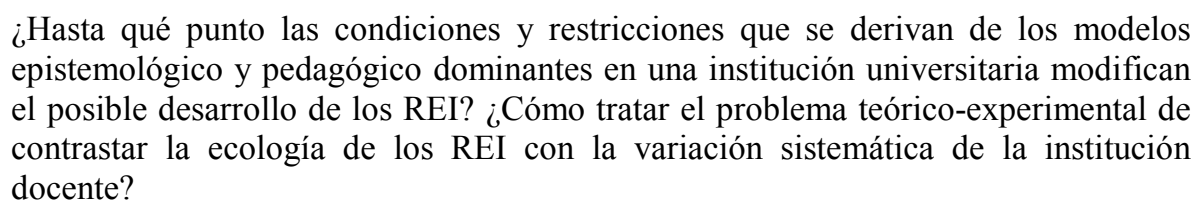

Consideramos, en este trabajo, una doble experimentación realizada a partir de un mismo REI impartido en dos instituciones universitarias distintas: en el grado de matemáticas en la Universidad de Copenhague (curso 2008/09) y en la Universidad Autónoma de

\footnotetext{
${ }^{1}$ Estas características corresponden (a grandes rasgos) a la caracterización del aplicacionismo como uno de los componentes principales de la epistemología dominante en la enseñanza universitaria de las matemáticas para las CCEE y que se pone de manifiesto en la forma concreta de interpretar, describir y conceptualizar la relación entre las matemáticas y las CCEE (BARQUERO; BOSCH; GASCON, 2014).
} 
Barcelona (curso 2009/10) ${ }^{2}$. Cabe destacar que en estos cursos nos encontramos en condiciones bastante ideales dadas de antemano: se tratan de cursos con estudiantes del grado universitario de matemáticas, que van a cursar una asignatura dedicada en exclusiva a la modelización matemática, la organización temporal de las sesiones son más largas que la organización de otros cursos (sesiones Taller de 2 horas, un par o tres días a la semana), el profesorado implicado en su docencia está muy formado en el ámbito de modelización, entre otros aspectos que iremos destacando a lo largo del trabajo.

\section{Diseño de un REI en torno al estudio de la evolución de la distribución de bicicletas}

A continuación, describimos el diseño de un REI a partir de una cuestión generatriz $Q_{0}$ en torno al estudio de la dinámica del flujo de bicicletas en la ciudad de Barcelona, sirviendo de hilo conductor de todo el proceso didáctico. Su estudio generará la construcción de una cadena de cuestiones y respuestas a partir de un proceso progresivo de modelización matemática y con una constante dialéctica con los medios disponibles, que nos llevará a la configuración del cuestión-grama ${ }^{3}$ del REI que nos servirá de base para analizar posteriormente sus respectivas implementaciones.

\subsection{La cuestión generatriz del proyecto Bicing}

Bicing $^{4}$ es el nombre de un servicio público de uso compartido de bicicletas que se inauguró en Barcelona (España), en 2007. Este servicio, no se trata de un sistema de uso turístico de bicicletas, sino que tiene como objetivo cubrir pequeñas y medianas rutas diarias de los ciudadanos de Barcelona. Dos años después de su inicio, con 200 bicicletas y 15 estaciones, las cifras desbordaron totalmente toda previsión posible, la red dispone actualmente de 400 estaciones (Figura 1) donde recoger y devolver una flota total de 6000 bicicletas distribuidas en todo el sistema.

\footnotetext{
${ }^{2}$ Cabe mencionar que en los cursos: 2012/13 y 2013/14 se ha desarrollado una variante de este mismo REI en una tercera institución universitaria, la del grado en administración y dirección de empresas de IQS-School of Management de la Universitat Ramon Llull en Barcelona (España).

${ }^{3}$ Con la expresión de cuestión-grama queremos referirnos, de forma simplificada, a la estructura arborescente de los REI la cual, en su descripción más extensa, incluiría no solo la evolución de las cuestiones (inicial y derivadas), sino también la dinámica y dialéctica que se establece entre las cuestiones derivadas, sus respuestas parciales y finales y la dialéctica entre los medios y media disponibles en cada punto del estudio (BARQUERO; BOSCH; GASCÓN, 2013).

${ }^{4}$ Para más información sobre Bicing se puede consultar su enlace principal: https://www.bicing.cat/
} 


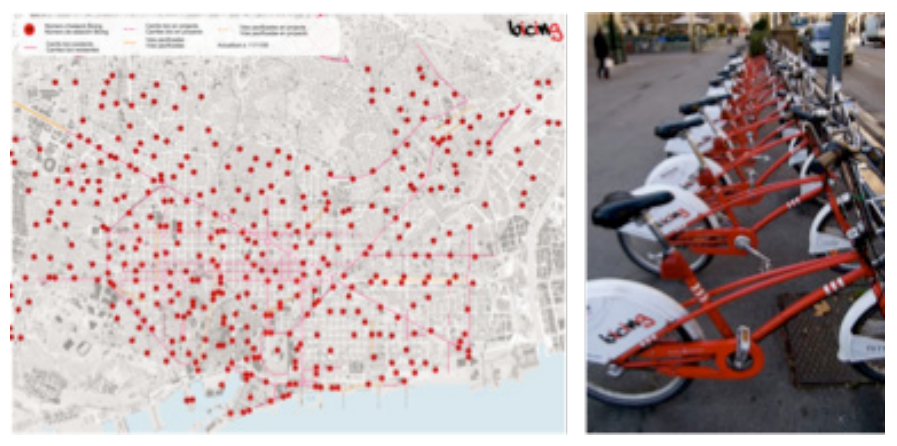

Figura 1 - Mapa de las estaciones de Bicing distribuidas en toda la ciudad.

Fuente: https://www.bicing.cat/sites/default/files/docs/Mapa-bicing-10-2014.jpg

El éxito inicial del sistema se vio rápidamente afectado por numerosas quejas: bicicletas averiadas, estaciones vacías en algunas zonas, anclajes insuficientes, robos de bicicletas eran, entre otros, algunos de los problemas más habituales. La complejidad y la búsqueda de posibles mejoras del sistema presenta muchas cuestiones que requieren un estudio profundizado. Los encargados de la gestión de Bicing solicitaron diversos estudios con el objetivo de que se describiera y estudiara qué cambios y mejoras se podían proponer en la distribución de bicicletas dentro del sistema. Nos proponen, así, centrarnos en estudiar la siguiente cuestión inicial (y generatriz) del estudio:

$\boldsymbol{Q}_{0}$ : Dada la demanda actual, ¿cómo podemos mejorar la distribución de bicicletas dentro del sistema?

El estudio de esta cuestión generatriz puede desglosarse en dos sub-cuestiones que corresponden a dos posibles fases del estudio:

$\boldsymbol{Q}_{\mathbf{0}(\mathrm{A})}$ : ¿Cómo podemos describir el flujo de bicicletas que hay diariamente entre las distintas estaciones? ¿Cuál sería la evolución natural del sistema si fuese auto-gestionado (sin reposición de bicicletas)?

$\boldsymbol{Q}_{\mathbf{0 ( B )}}:$ ¿Cómo podemos predecir las necesidades de reposición de bicicletas? ¿Qué cambios se deberían introducir para que el sistema tuviese más capacidad de mover el material necesario en el menor tiempo posible? ${ }^{5}$

Después de un primer análisis del tráfico de bicicletas entre las distintas estaciones que la empresa nos facilita, los diseñadores (e investigadores) deciden agrupar las estaciones por áreas o zonas de la ciudad según el patrón de comportamiento de estas. Finalmente, se decide considerar seis zonas principales (ver Figura 2). Además, a partir de los datos que Bicing nos proporciona, se puede construir la siguiente tabla que representa la matriz origen-destino (matriz OD) que indica el número potencial de viajes diarios ${ }^{6}$. Los datos se obtuvieron

\footnotetext{
${ }^{5}$ En este trabajo nos centramos en describir el posible desarrollo de la cuestión $Q_{0 \text { (A) }}$, aunque es importante enunciar $Q_{0(\mathrm{~B})}$ que representa la segunda fase del estudio derivada de $Q_{0}$.

${ }^{6}$ Consideramos como diario la franja en que el servicio Bicing está activo, esta es, de domingo a jueves de las $5 \mathrm{~h}$. de la mañana a medianoche y $24 \mathrm{~h}$. los fines de semana.
} 
después de un estudio, durante varios meses, de la demanda de los usuarios de Bicing. Cada dato de la tabla representa el promedio del número total de viajes realizados diariamente que parten de una determinada zona (columnas $j$ ) y llegan a otra al final del día, o se quedan en la misma zona (filas $i$ ).

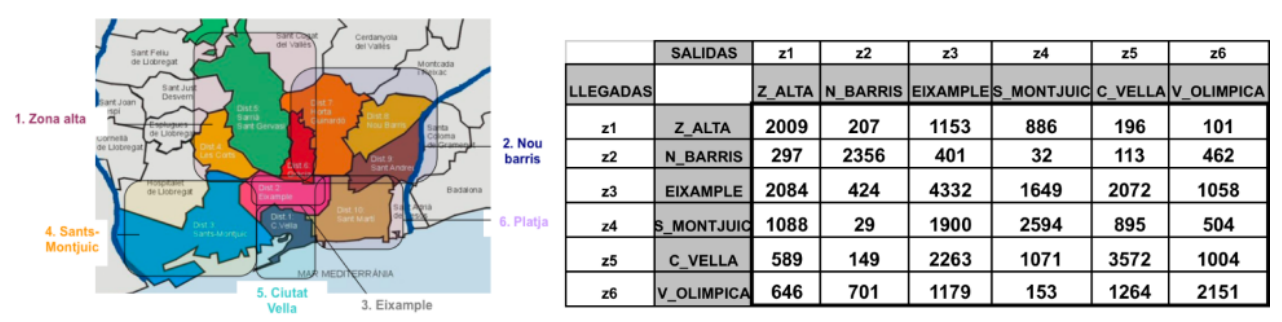

Figura 2 - Agrupación de las estaciones en las seis zonas y matriz OD de viajes diarios

Si denotamos con $s(k)$ el número total de salidas que ha tenido una zona $k$ al inicio de funcionamiento del sistema y como $l(k)$ la cantidad de llegadas en esta misma zona al final de la jornada, podemos empezar por analizar, por ejemplo, la tasa absoluta variación: $l(k)-s(k)$ para tener una primera aproximación de las zonas que diariamente tienen déficit o exceso de bicicletas.

Aunque para avanzar en el estudio de la cuestión $Q_{0(\mathrm{~A})}$ debemos ir más allá del estudio numérico-descriptivo de los datos. Esto nos conducirá a la construcción de modelos basados en sucesiones recurrentes de orden $d>1$ reducibles a sucesiones recurrentes vectoriales del tipo $X_{n+1}=f\left(X_{n}\right)$ donde $X_{n}$ es el vector de la distribución de bicicletas en las distintas zonas en tiempo $n$. El estudio de $Q_{0 \text { (A) }}$ nos llevará a la construcción de este tipo de modelos matriciales y, en función de la formulación de hipótesis que describiremos a continuación, nos conducirá al problema del cálculo de la potencia $n$-ésima de una matriz.

\subsection{Del análisis descriptivo de la matriz OD a las matrices de transición}

Si suponemos que en el instante inicial $(n=0)$ conocemos cuál es la distribución inicial de bicicletas en las distintas zonas que tenemos predefinidas, podemos denotar por $X(0)$ al vector cuya componente $x_{i}(0)$ corresponde al número de bicicletas que hay en este instante inicial en la zona $i$. Así, $X(0)=\left(x_{1}(0), x_{2}(0), \ldots, x_{6}(0)\right)$ contiene la distribución inicial de bicicletas en las seis zonas predefinidas. Para estudiar la evolución de la distribución de bicicletas, nos proponemos estudiar cómo evolucionará el vector: $X(n)=\left(x_{1}(n), x_{2}(n), \ldots, x_{6}(n)\right)$ que contiene la distribución del total de bicicletas en tiempo $n$. 
$\boldsymbol{H}_{(\mathbf{A}) 1}$ : Podemos suponer que no hay reposición (ni desaparición) de bicicletas en el sistema y que el tráfico de bicicletas entre las estaciones cada día se mantiene el mismo.

$\boldsymbol{Q}_{(\mathbf{A}) 1.1}$ : Si por la mañana, al iniciarse el sistema, situamos diferentes distribuciones iniciales de bicicletas en cada estación, ¿cuántas bicicletas habrá al final del día? ¿Cuál será la distribución de bicicletas después de $2,3,4, \ldots, 30$ días etc.?

Ante el problema de previsión descrito por $Q_{(\mathrm{A}) 1.1} \mathrm{y}$, a partir de la formulación de las primeras hipótesis, podemos construir un primer modelo matemático $\left(M_{1}\right)$. A partir de la información que nos proporciona la matriz OD (ver Figura 3) se puede calcular el porcentaje de transición entre dos zonas, como: el número potencial de viajes diarios que salen de $j$ y llegan a $i\left[\mathrm{OD}_{i j}\right]$ dividido entre el total de salidas en la zona $j[s(j)]$, pudiéndolo calcular para toda componente de la matriz OD:

\begin{tabular}{|c|c|c|c|c|c|c|c|}
\cline { 2 - 8 } \multicolumn{1}{c|}{} & SALIDAS & $\mathrm{z} 1$ & $\mathrm{z2}$ & $\mathrm{z3}$ & $\mathrm{z4}$ & $\mathrm{z} 5$ & $\mathrm{z} 6$ \\
\hline LLEGADAS & & Z_ALTA & N_BARRIS & EIXAMPLE & S_MONTJUIC & C_VELLA & V_OLIMPICA \\
\hline $\mathrm{z} 1$ & Z_ALTA & 2009 & 207 & 1153 & 886 & 196 & 101 \\
\hline $\mathrm{z} 2$ & N_BARRIS & 297 & 2356 & 401 & 32 & 113 & 462 \\
\hline $\mathrm{z} 3$ & EIXAMPLE & 2084 & 424 & 4332 & 1649 & 2072 & 1058 \\
\hline $\mathrm{z} 4$ & S_MONTJUII & 1088 & 29 & 1900 & 2594 & 895 & 504 \\
\hline $\mathrm{z} 5$ & C_VELLA & 589 & 149 & 2263 & 1071 & 3572 & 1004 \\
\hline $\mathrm{z} 6$ & V_OLIMPICA & 646 & 701 & 1179 & 153 & 1264 & 2151 \\
\hline
\end{tabular}

\begin{tabular}{|c|c|c|c|c|c|c|}
\cline { 2 - 7 } \multicolumn{1}{|c|}{} & $z 1$ & $z 2$ & $z 3$ & $z 4$ & $z 5$ & $z 6$ \\
\hline$z 1$ & 0,3 & 0,1 & 0,1 & 0,1 & 0,0 & 0,0 \\
\hline$z 2$ & 0,0 & 0,6 & 0,0 & 0,0 & 0,0 & 0,1 \\
\hline$z 3$ & 0,3 & 0,1 & 0,4 & 0,3 & 0,3 & 0,2 \\
\hline$z 4$ & 0,2 & 0,0 & 0,2 & 0,4 & 0,1 & 0,1 \\
\hline$z 5$ & 0,1 & 0,0 & 0,2 & 0,2 & 0,4 & 0,2 \\
\hline$z 6$ & 0,1 & 0,2 & 0,1 & 0,0 & 0,2 & 0,4 \\
\hline Suma & 1,00 & 1,00 & 1,00 & 1,00 & 1,00 & 1,00 \\
\hline
\end{tabular}

Figura 3 - Ejemplificación de la transformación de la tabla o matriz OD a la matriz de transición

La matriz resultante es, en realidad, la denominada matriz de transición asociada a la matriz OD cuyos elementos $M=\left(m_{i j}\right)$ son las probabilidades de transición de que una bicicleta situada en la $j$-ésima zona en el instante $t=n$, en el instante siguiente $t=n+1$ haya cambiado a la $i$-ésima zona. Una vez introducida la notación necesaria con la que vamos a trabajar y las propiedades básicas de las matrices de transición, podemos describir la evolución de la distribución de bicicletas en periodos de tiempo consecutivos con la siguiente ecuación o relación matricial: $X(n)=M \cdot X(n-1)$. Si suponemos, además, que los porcentajes de transición se mantienen constantes con el paso del tiempo $n$, entonces la relación descrita equivale a la expresión:

$$
X(n)=M \cdot X(n-1) \leftrightarrow X(n)=M^{n} \cdot X(0) \text { para } n>0
$$

Con el uso de este primer modelo podemos dar respuesta a $Q_{(\mathrm{A}) 1.1}$ a través de la simulación de la trayectoria de $X(n)$ con cualquiera de les expresiones equivalentes descritas en (1). A partir de las simulaciones realizadas con diferentes distribuciones iniciales de bicicletas, se pueden plantear nuevas cuestiones y respuestas:

$\boldsymbol{Q}_{(\mathbf{A}) 1.2}$ : Dada la matriz de transición $M$ y distintas distribuciones iniciales $X(0)$, ¿qué características podemos destacar de la trayectoria de la sucesión vectorial $X(n)$ definida por (1)?

$\boldsymbol{R}_{(\mathrm{A}) 1.2}$ : Sea $M$ una matriz de transición y $X(0)$ la distribución inicial de bicicletas dentro del sistema, la trayectoria de la sucesión $X(n)$ parece siempre tender a un vector fijo o distribución estable o de equilibrio que podemos denotar por $X^{\mathrm{e}}$. 
Más detalles sobre el desarrollo matemático de esta posible rama del REI se pueden encontrar en Fonseca (2010) y Barquero (2009, p.138-167). Esta primera simulación numérica, que conduce a la detección de la existencia de distribuciones estables, nos permite formular nuevas hipótesis referentes al sistema que estamos estudiando y seguir formulando cuestiones cada vez más matematizadas:

$\boldsymbol{Q}_{(\mathrm{A}) 1.3}$ : ¿Cómo podemos demostrar la existencia de vectores fijos $X^{\mathrm{e}}$ hacia los cuales parece tender toda trayectoria de $X(n)$ generada por la ecuación matricial (1) con $M$ una matriz de transición? ¿Toda trayectoria de $X(n)$ converge a este vector fijo $X^{\mathrm{e}}$ ? En caso que existan, ¿cómo podemos calcular $X^{\mathrm{e}}$ a priori (sin necesidad de simularlo numéricamente)? ¿Cuál es la dependencia entre $X^{\mathrm{e}}, M$ y $X(0)$ ?

$\boldsymbol{Q}_{(\mathbf{A}) 1.4}:$ ¿Qué relación hay entre $X^{e}$ y las propiedades de la potencia $n$-ésima de la matriz $M$ ?

E incluso cuestiones que conducirán a detectar las limitaciones de este primer modelo, a reformular las hipótesis y a la construcción de un segundo modelo:

$\boldsymbol{Q}_{(\mathbf{A}) 1.5}:$ ¿Tiene sentido suponer que el sistema siempre actúa igual independientemente de, por ejemplo: los viajes que hace una bicicleta por día, la demanda potencial de bicicletas, el total de bicicletas disponibles, etc.?

\subsection{Hacia un modelo con restricciones sobre la demanda y la disponibilidad de bicicletas}

En la construcción del primer modelo, se está asumiendo, implícitamente, diversas características muy importantes: (1) en cada cálculo del iterado $X(n+1)$ respecto $X(n)$ representa que ha transcurrido todo un día de servicio y, (2) una transición significa la probabilidad de cambio de posición (de una zona a otra, o en la misma), cuando una bicicleta hace solamente un viaje. Al explicitar estas hipótesis es cuando vemos que no tiene mucho sentido, la matriz OD ya nos decía que había un número de viajes diarios bastante superior al número total de bicicletas de las que dispone el sistema (un total de 41584 viajes diarios con una flota cerca de 6000 bicicletas). Por ello, nos planteamos una nueva cuestión que nos lleva a la reformulación de las hipótesis y, con ello, a la construcción y desarrollo de un modelo más completo y complejo:

$\boldsymbol{Q}_{(\mathrm{A}) 2}$ : ¿Cómo podemos describir la evolución del flujo de bicicletas cada 30 minutos, añadiendo el hecho de que no todas las bicicletas (6000 en total) no se mueven en cada franja de 30'?

$\boldsymbol{H}_{(\mathbf{A}) 2}$ : Vamos a suponer que: (1) cada viaje de una bicicleta es de 30 '; (2) El total de la flota de bicicletas (6000 en total) no se mueve cada fracción de 30'; (3) El número total de bicicletas que finalmente se mueve en el instante de tiempo $t$ va a depender de: (a) la demanda potencial de bicicletas cada 30', y (b) el total de bicicletas disponibles en el instante de tiempo $t$.

Vamos a definir que nuestra variable tiempo $t$ describe las sucesivas franjas de 30 minutos (tiempo promedio de uso de una bicicleta dentro del sistema) que tiene una jornada 
de servicio de Bicing. Si definimos $B_{i}(t)$ como el número de bicicletas que hay en cada zona en la franja de tiempo $t$, podemos definir el vector total de bicicletas como $B(t)=\left(B_{1}(t), B_{2}(t), B_{3}(t), B_{4}(t), B_{5}(t), B_{6}(t)\right)$. Si definimos además $S_{i}(t)$ como la cantidad de salidas de bicicletas de la zona $i$ en tiempo $t$, podemos describir el vector salidas como $S(t)=\left(S_{1}(t), S_{2}(t), S_{3}(t), S_{4}(t), S_{5}(t), S_{6}(t)\right) \mathrm{y}$, finalmente, si definimos $L_{i}(t)$ como la cantidad de llegadas de bicicletas a la zona $i$, el vector llegadas es $L(t)=\left(L_{1}(t), L_{2}(t), L_{3}(t), L_{4}(t), L_{5}(t), L_{6}(t)\right)$. Entonces, el total de bicicletas en cada zona en tiempo $t$ puede estimarse como:

$$
B(t+1)=B(t)-S(t)+L(t+1)
$$

Pero, ¿cómo podemos predecir el total de salidas que habrá en cada zona $S(t)$, y el total de llegadas en la franja de tiempo siguiente $L(t+1)$ ?

De acuerdo con $H_{(\mathrm{A}) 2}$, podemos asumir que el número total de bicicletas que finalmente se mueve en el instante de tiempo $t$ depende de: (a) la demanda potencial de bicicletas cada fracción de 30' [demanda_viajes(30’)] y (b) del total de bicicletas disponibles en el instante de tiempo $t$. El total de salidas se puede definir entonces como:

$$
S(t)=\min \left(\text { demanda_viajes }\left(30^{\prime}\right), B(t)\right)
$$

donde para calcular la demanda potencial de bicicletas cada franja de 30' debemos utilizar la información de la matriz OD que nos describe la demanda potencial diaria de bicicletas. Debemos, así, añadir nuevas suposiciones que, entre todas las posibles, la más sencilla es la de suponer que esta demanda se distribuye uniformemente a lo largo de todas las franjas de 30' de un día de servicio. Esta suposición nos lleva a tener que dividir los datos de la matriz OD entre tantas franjas de 30' como tenga un servicio de Bicing para estimar la demanda potencial de cada franja de tiempo ${ }^{7}$.

Por otro lado, el total de llegadas se define como:

$$
L(t+1)=M \cdot S(t)
$$

donde $\mathrm{M}$ es una la matriz de transición con las probabilidades de transición (cada 30') que una bicicleta se traslade de una zona $i$ a $j$. Esta matriz de transición es exactamente la misma que en el primer modelo (y sería exactamente la misma aunque quisiéramos describir las transiciones en intervalos de tiempo más pequeños).

\footnotetext{
${ }^{7}$ Aquí podrían aparecer numerosas modificaciones y ampliaciones de estas hipótesis. Se está describiendo el caso más sencillo, una distribución uniforme de la demanda, pero podríamos pensar en suposiciones más complejas: la existencia de horas punta en las que la carga de flujo fuese superior al promedio o en la necesidad de considerar diferentes matrices origen-destino a lo largo de día, etc.
} 
Una vez construido este segundo modelo, podemos plantear muchas nuevas cuestiones para guiar su proceso de estudio:

$\boldsymbol{Q}_{(\mathbf{A}) 2.1}$ : Usando este segundo modelo, y dando diferentes distribuciones iniciales de bicicletas $B(0)$, ¿cuál será la distribución de bicicletas después de $2,3,4, \ldots, 30$ días etc.?

$\boldsymbol{Q}_{(\mathbf{A}) 2.2}:$ ¿Qué características podemos destacar de la trayectoria de la sucesión vectorial $B(t)$ definida por la ecuación matricial (2)? ¿Existen también vectores fijos o distribuciones estables?

$\boldsymbol{Q}_{(\mathrm{A}) 2.3}:$ ¿Qué relación hay entre $n$-ésima de la matriz $M$ y la posible convergencia de $B(t)$ ? etc.

$\boldsymbol{Q}_{(\mathrm{A}) 2.4}$ : ¿Qué relación hay entre el primer modelo y el segundo? ¿Y a las conclusiones a las que se llegan? ¿Hasta qué punto el segundo modelo llega a superar limitaciones que se habían destacado del primero? ¿Podemos mejorar en algún sentido los modelos construidos? etc.

Cabe destacar que el primer modelo $\left(M_{1}\right)$ es un caso particular de este segundo modelo $\left(M_{2}\right)$, es decir, si en este segundo modelo consideramos que siempre hay suficientes bicicletas en el sistema para cubrir la demanda de los usuarios, entonces nos restringiríamos al estudio $\operatorname{con} M_{1}$.

En la siguiente Figura 5 se describe, a modo resumen, la propuesta del posible desarrollo de la cuestión inicial $Q_{0(\mathrm{~A})}$ :

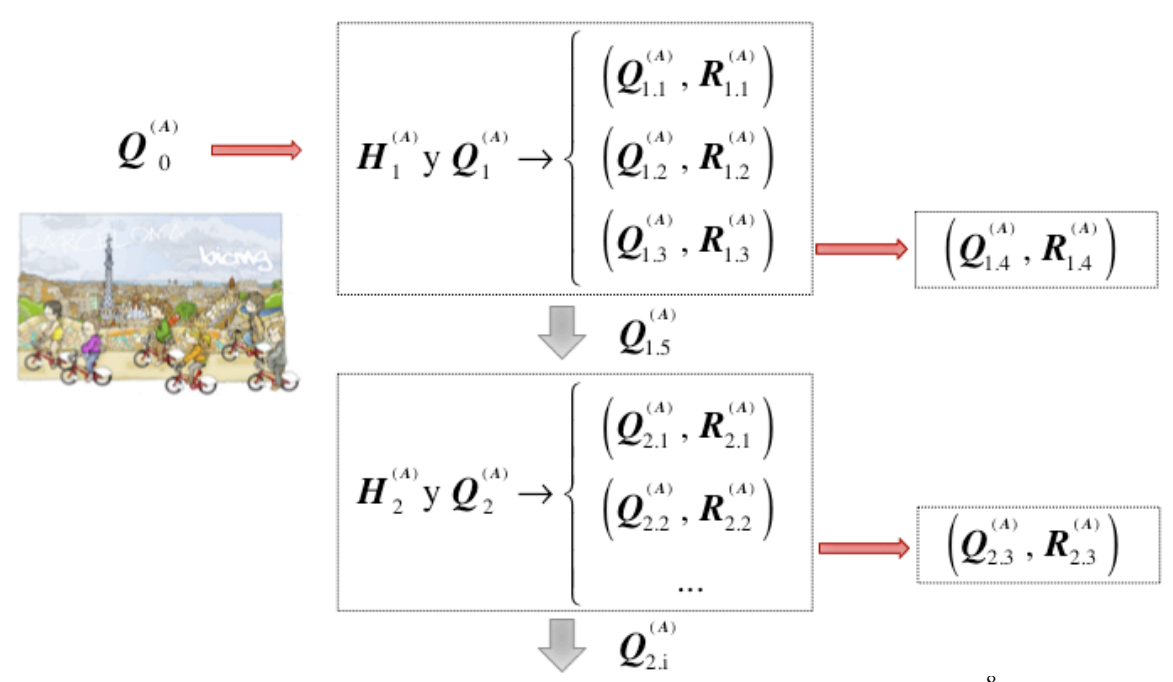

Figura 5 - Cuestión-grama del posible desarrollo de $Q_{(\mathrm{A}) 0}{ }^{8}$

\section{Experimentación, evaluación y desarrollo de un REI a nivel universitario}

La experimentación del proyecto Bicing se realizó en dos ocasiones, durante el curso 2008/09, con estudiantes del grado de matemáticas. Ambas experimentaciones se realizaron bajo condiciones que podrían considerarse excepcionales (en comparación a las descritas en BARQUERO; BOSCH; GASCÓN, 2011, 2014): nos situamos en asignaturas con estudiantes

\footnotetext{
${ }^{8}$ Las cuestiones $Q_{(\mathrm{A}) 1.4}$ y $Q_{(\mathrm{A}) 2.3}$ aparecen separadas del resto ya que son origen de la construcción y desarrollado de una organización matemática en torno a la diagonalización de matrices y al cálculo de sus potencias $n$-ésimas (ver más detalles en BARQUERO, 2009).
} 
del grado de matemáticas dedicadas exclusivamente a la enseñanza de la modelización matemática. Muchas de las restricciones que habíamos tenido que aceptar en investigaciones previas quedan, aquí, muy atenuadas: la rigidez de los dispositivos didácticos tradicionales teoría-ejercicios-examen, el papel secundario otorgado a la modelización, el examen final como único dispositivo de evaluación, la estructura tradicional de los programas en términos de contenidos matemáticos, entre otras. Aunque debemos destacar, antes de empezar con la breve descripción de ambas experimentaciones, que los cursos plantearon propuestas docentes bastante antagónicas.

\subsection{Primera experimentación: el Bicing project en la Universidad de Copenhague}

La primera de las experimentaciones se realizó en la Universidad de Copenhague (UC) con un total de 23 estudiantes de 3 r curso del grado de matemáticas que participaban en el curso optativo llamado MathMod. Este curso se concentraba en 7 semanas de clase, más dos semanas adicionales al final para preparar el proyecto final de la asignatura. Cada semana se realizaban tres sesiones de dos horas que, en general: la primera siempre se dedicaba a una clase teórica por parte del profesor, la siguiente a la resolución de algunos ejercicios prácticos y la tercera a trabajo en grupo, normalmente en la sala de informática y trabajando con Mapple, para avanzar en las distintas entregas del curso. La propuesta docente del curso se basaba en la realización de cuatro mini-proyectos, junto con algunos listados adicionales de problemas. Estos proyectos consistían, principalmente, en poner en prácticas algunos modelos bastante conocidos en torno al estudio de la dinámica de poblaciones que en la sesión inaugural de teoría se presentaban como ya construidos, por ejemplo: Mini-proyectol Predicción del crecimiento de una población con los modelos maltusiano y logístico o Miniproyecto4-Modelos predador-presa.

Aprovechando la estancia predoctoral que la autora de este trabajo realizó en la UC, el profesor encargado del curso ofreció la generosa posibilidad de incorporar un quinto proyecto que sería, en este caso, el Bicing project. Este ocupó, finalmente, dos semanas completas (6 sesiones de 2 horas), combinando presentaciones por parte de la profesora-investigadora o de los grupos de estudiantes, sesiones de trabajos en grupos y sesiones en la sala de informática. Al finalizar cada bloque de tres sesiones, cada grupo tuvo que entregar un informe con la síntesis de su trabajo realizado. 


$$
\left.\begin{array}{c}
\boldsymbol{Q}_{0}^{(A)} \Longrightarrow\left[\begin{array}{c}
\left(\boldsymbol{Q}_{1.1}^{(A)}, \boldsymbol{R}_{1.1}^{(A)}\right) \\
\left(\boldsymbol{Q}_{1.2}^{(A)}, \boldsymbol{R}_{1.2}^{(A)}\right) \\
\left(\boldsymbol{Q}_{1.3}^{(A)}, \boldsymbol{R}_{1.3}^{(A)}\right)
\end{array}\right] \Longrightarrow\left(\boldsymbol{Q}_{1.4}^{(A)}, \boldsymbol{R}_{1.4}^{(A)}\right) \\
\boldsymbol{Q}_{1.5}^{(A)}
\end{array}\right]
$$

Figura 6 - Resumen del cuestión-grama en el caso de la experimentación en la UC

Durante la primera semana, una vez presentada la $Q_{0}$ y centrándonos primeramente en $Q_{0(\mathrm{~A})}$, se desarrolló casi todo el estudio referente a la construcción del primer modelo basado en matrices de transición (descrito en el apartado 3.2). Con la gran ventaja que en el segundo mini-projecto ya habían estudiado las propiedades de la potencia $n$-ésima de las matrices de Leslie y de las matrices de transición, hecho que facilitó mucho el estudio de $Q_{(\mathrm{A}) 1.3}$ y $Q_{(\mathrm{A}) 1.4}$.

La segunda semana, nos centramos en explicitar las limitaciones del primer modelo y reformular las nuevas hipótesis y cuestiones: $H_{(\mathrm{A}) 2}$ y $Q_{(\mathrm{A}) 2}$, aunque no se pudo avanzar mucho más allá de la construcción del segundo modelo, por limitaciones de tiempo, y solo se trató

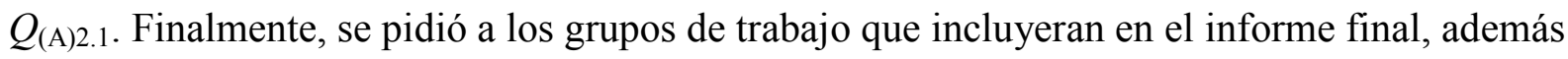
del trabajo realizado, algunas sugerencias a Bicing de cómo mejorar su política de distribución de bicicletas, $Q_{0(\mathrm{~B})}$.

\subsection{Segunda experimentación: primera adaptación del REI}

Unos meses después se realizó la segunda experimentación del REI en la Universitat Autònoma de Barcelona (UAB). Por primera vez se inauguraba una asignatura obligatoria en el grado de matemáticas llamada: Taller de modelización matemática de $2^{\circ}$ curso, $2^{\circ}$ semestre. En esta primera edición del curso participaron un total de 45 estudiantes. En este caso, la propuesta docente del curso era completamente diferente. La principal componente del curso es el desarrollo de un proyecto que los estudiantes, distribuidos en grupos, escogen por votación durante una sesión del curso. Esta tarea es supervisada por los profesores aunque, en general, nunca intervienen de forma directa en el desarrollo del proyecto. Paralelamente, se intercalan algunos ejemplos para desarrollar las ideas generales sobre modelización matemática. Durante el curso 2008/09, hubo dos profesores encargados del curso (uno de los cuales es la autora del trabajo). El proyecto Bicing sirvió para inaugurar el curso y acabó por 
ocupar 5 semanas del curso, con dos sesiones de dos horas por semana. Se pidió que los estudiantes, distribuidos en grupos de cuatro o cinco personas, entregaran semanalmente un informe parcial con la síntesis del trabajo realizado (en base a las cuestiones abordadas, herramientas y modelos matemáticos considerados, respuestas encontradas y nuevas cuestiones para seguir con el estudio) y, al terminar el proyecto, un informe final como síntesis de todo el estudio desarrollado.

El proceso de estudio generado a partir de $Q_{0(\mathrm{~A})}$ no se diferencia excesivamente del que se desarrolló en la primera de las experimentaciones, aunque en esta ocasión no se entró a hacer ninguna propuesta sobre $Q_{0(\mathrm{~B})}$, ni tampoco se plantearon ni estudiaron las cuestiones relativas a las propiedades de las potencia $n$-ésimas de las matrices de transición involucradas: $Q_{(\mathrm{A}) 1.4}$ ni $Q_{(\mathrm{A}) 2.3}$ ya que simplemente los estudiantes no las plantearon dentro de sus propuestas. Una novedad importante fue que los estudiantes plantearon si la empresa disponía de matrices OD más segmentadas, por ejemplo, distinguiendo el comportamiento del flujo entre mañana y tarde. Los estudiantes habían estado analizando el sistema real y habían podido constatar que Bicing tenía comportamientos diferentes, como mínimo, en estas dos franjas horarias. Así que se solicitó a la empresa y se les entregó dos nuevas matrices origendestino, basadas en datos reales, para distinguir los distintos comportamientos de la mañana (05:00h-14:30h) y la tarde (14:30h-00:00h). De esta forma, el estudio desarrollado en esta experimentación se extendió hacia la construcción de un tercer modelo $M_{3}$ que, construido en base a los dos modelos anteriores $M_{1}$ y $M_{2}$, distinguía la matriz OD utilizada según la franja horaria.

$$
\begin{gathered}
\boldsymbol{Q}_{0}^{(A)} \Longrightarrow\left[\begin{array}{c}
\left(\boldsymbol{Q}_{1.1}^{(A)}, \boldsymbol{R}_{1.1}^{(A)}\right) \\
\left(\boldsymbol{Q}_{1.2}^{(A)}, \boldsymbol{R}_{1.2}^{(A)}\right) \\
\left(\boldsymbol{Q}_{1.3}^{(A)}, \boldsymbol{R}_{1.3}^{(A)}\right)
\end{array}\right] \\
\boldsymbol{H}_{1}^{(A)} \text { у } \boldsymbol{Q}_{1}^{(A)} \boldsymbol{Q}_{1.5}^{(A)} \\
\boldsymbol{H}_{2}^{(A)} \text { у } \boldsymbol{Q}_{2}^{(A)} \rightarrow\left\{\begin{array}{c}
\left(\boldsymbol{Q}_{2.1}^{(A)}, \boldsymbol{R}_{2.1}^{(A)}\right) \\
\left(\boldsymbol{Q}_{2.2}^{(A)}, \boldsymbol{R}_{2.2}^{(A)}\right)
\end{array}\right] \\
\boldsymbol{H}_{3}^{(A)}, \boldsymbol{Q}_{3}^{(A)} \text { у } \boldsymbol{M}_{3}^{(A)}
\end{gathered}
$$

Figura 7 - Resumen del cuestión-grama en el caso de la experimentación en la UAB 


\section{Conclusiones sobre la relatividad institucional de los REI}

A pesar de que ambas experimentaciones fueron muy ricas y se ajustaron bastante a su diseño previsto, tanto los estudiantes como los profesores encargados de los cursos manifestaron diversas resistencias que sacaron a la luz algunas restricciones importantes. En lo que se refiere a los estudiantes las reacciones fueron bastante diferentes, debidas, sin duda, a una diferencia esencial entre ambas experimentaciones: en la UC la experimentación del REI se realizó al final del curso MathMod en el que el contrato didáctico ya estaba bien establecido y los estudiantes ya tenían cierta práctica de lo que, según el enfoque del curso, significaba desarrollar una actividad de modelización matemática. Esperaban, por lo tanto, que fuera el profesor quien propusiera los modelos que había que utilizar y los problemas y sistemas a estudiar. Pero ya disponían de un vocabulario para referirse a la actividad de modelización, habían cogido el hábito de trabajar en grupo, de redactar informes, discutirlos, defenderlos etc. Por el contrario, en la UAB, la experimentación tuvo lugar al inicio del curso y los estudiantes manifestaron muchas de las resistencias que eran predecibles al toparse con unas propuestas completamente atípicas dentro de unos estudios universitarios de matemáticas, resistencias que se debían, principalmente, a cambios bruscos en el contrato didáctico tradicional y a la nueva organización didáctica del curso.

Si nos centramos en la primera experimentación en la UC, podemos considerar que algunas de las restricciones que surgieron, específicamente aquí, fueron consecuencia (o manifestación) del impacto que tiene el modelo epistemológico espontáneo subyacente en las posibles formas de desarrollar el conjunto de prácticas de modelización matemática. Creemos que dicho modelo epistemológico comparte fuertes rasgos aplicacionistas (BARQUERO; BOSCH; GASCÓN, 2014), por ejemplo, el asumir una distinción neta entre las matemáticas y sus posibles intrusiones en los sistemas extra-matemáticos, donde las primeras preexisten a los últimos. En esta concepción, la actividad de modelización se restringe a la ejemplificación puntual de ciertos modelos preestablecidos. Como consecuencia, la estructura y dinámica del curso siempre presentaba una misma distribución: una primera sesión en la que se el profesor enseñaba los modelos ya definitivos que, posteriormente, se aplicarían a distintas situaciones extra-matemáticas; el ejercicio del uso de estos modelos en casos prototípicos sencillos; finalmente, un uso más complejo en proyectos para realizar en grupo. En otras palabras, el modelo docente subyacente tiende, en coherencia con el modelo epistemológico espontáneo aplicacionista, a potenciar un encuentro cultural-mimético (CHEVALLARD, 1999) con los modelos previamente existentes. 
En este contexto, no es de extrañar que, al iniciarse el proyecto Bicing, los estudiantes de la UC presentaran mucha resistencia a tener que asumir nuevas responsabilidades que, hasta el momento, habían quedado en manos del profesor: formulación de hipótesis, construcción de modelos imperfectos (no cristalizados), uso de los modelos para dar respuestas a cuestiones, planteo de nuevas cuestiones, limitaciones y evolución de los modelos iniciales etc. Ahora bien, a pesar del desconcierto en las primeras sesiones, y del intento fracasado de utilizar todos los modelos que tenían en sus apuntes (maltusiano, logístico, predador-presa etc.) y ver que nada funcionaba, poco a poco los estudiantes fueron entrando en el desarrollo de su propio estudio.

Centrándonos, ahora, en la segunda de las experimentaciones, la de la UAB, en este caso las restricciones más claras no fueron manifestadas por los estudiantes, sino por el profesor responsable del curso. A pesar de que dejó total libertad a la investigadora para organizar las sesiones, en sus comentarios expresaba que el proceso seguido durante la experimentación del REI había sido muy largo, demasiado guiado, con demasiadas entregas y puestas en común y que se debía dejar más tiempo a los estudiantes para que trabajaran solos, sin interferir demasiado en su trabajo y dejarles explicar su propuesta final. Reacciones bastante contrarias a las presentadas en la experimentación anterior, donde el profesor danés consideraba que el problema era demasiado abierto y los estudiantes pedían constantemente ayuda sobre qué modelos utilizar.

En el caso de la UAB, podemos decir que la organización didáctica que el profesor quería implementar, y que describía mediante sus comentarios, corresponde a un modelo docente espontáneo que tiene un fuerte componente modernista (GASCÓN, 2001). Este modelo es compatible con un modelo epistemológico que, entre otras características, considera la creación como un proceso individual o, en todo caso, privado, y tiende a hacer desaparecer los espacios compartidos donde se pueda construir, cuestionar y acordar colaborativamente herramientas y estrategias para desarrollar el propio proceso de modelización. En este modelo, y como la propuesta del curso completo puso posteriormente en evidencia, la actividad de modelización matemática tiende a ser interpretada como el estudio y resolución de un conjunto de problemas aislados y suficientemente abiertos como para requerir una elaboración creativa informal, privada e inenarrable. Se tiende, así, a eliminar todos aquellos dispositivos didácticos que puedan dar espacio a un trabajo compartido sobre la propia actividad de creación y contraste de los modelos. Tampoco aparecen, de forma institucionalizada, muchas de las nociones, conceptos, discursos elementos de la tecnología matemática, en el sentido de la TAD - necesarios para poder 
hablar del trabajo de modelización, como las propias nociones de sistema, modelo, hipótesis que modifican el sistema etc.) y poder institucionalizarlo. Todo ocurre como si, en el contrato didáctico establecido, el proceso de construcción de los modelos y de las respuestas derivadas del trabajo con estos modelos quedara en la esfera de lo privado y fuera del trabajo compartido entre el profesor y los alumnos en el aula; como si el propio contrato se encargara de ocultar lo didáctico, haciendo invisible el proceso de producción de conocimientos matemáticos nuevos.

El problema de la ecología de la modelización matemática, y su problema correlativo de la introducción y difusión de los dispositivos didácticos necesarios para sustentar nuevas organizaciones didácticas para una enseñanza funcional de las matemáticas, en nuestro caso, la propuesta de los REI requiere, hoy en día, grandes esfuerzos e investigación para pasar de un estadio experimental a una práctica generalizada. Nos encontramos frente a una gran aventura que va a requerir generar nuevas condiciones que superen restricciones que son muy difíciles de abordar, puesto que se enraízan en las concepciones más profundas de qué son las matemáticas, en qué consiste el proceso de construcción de matemáticas nuevas y qué condiciones facilitan el desarrollo de este proceso. Todo un desafío que seguro sobrepasa el ámbito de actuación de la TAD, e incluso el ámbito de actuación de la didáctica de las matemáticas en general, requiriendo la cooperación de toda la comunidad escolar.

\section{Referencias}

ARTAUD, M. Some Conditions for Modelling to Exist in Mathematics Classrooms. In: BLUM, W.; GALBRAITH, P. L.; HENN, H-W.; NISS, M. (Eds.). Modelling and applications in mathematics education: The 14th ICMI study. New York: Springer, 2007. p. 371-377.

BARQUERO, B. Ecología de la modelización matemática en la enseñanza universitaria de las matemáticas. 2009. 564p. Tesis Doctoral (Doctoral en Matemáticas) - Facultad de Ciencias, Departamento de Matemáticas, Universidad Autónoma de Barcelona, Barcelona, España, 2009.

BARQUERO, B.; BOSCH, M.; GASCÓN, J. Los Recorridos de Estudio e Investigación y la modelización matemática en la enseñanza universitaria de las Ciencias Experimentales. Enseñanza de las Ciencias. Revista de investigación y experiencias didácticas, Bellaterra, Barcelona, v. 29, n. 3, p. 339-352. Noviembre. 2011.

BARQUERO, B.; BOSCH, M.; GASCÓN, J. The ecological dimension in the teaching of mathematical modelling at university. Recherches en Didactique de Mathématiques (RDM), Grenoble, v. 33 n. 3, p. 307-338. Diciembre. 2013.

BARQUERO, B.; BOSCH, M.; GASCÓN, J. Incidencia del 'aplicacionismo' en la integración de la modelización matemática en la enseñanza universitaria de las ciencias experimentales. Enseñanza de las Ciencias. Revista de investigación y experiencias didácticas, Bellaterra, Barcelona, v. 32, n. 1, p. 83-100. Marzo. 2014. 
BURKHARDT, H. Modelling in Mathematics Classrooms: reflections on past developments and the future. ZDM: The International Journal on Mathematics Education, Springer Berlin Heidelberg, v. 38, n. 2, p. 178-195. Marzo. 2006.

CASTELA, C. Institutions influencing mathematics students' private work: a factor of academic achievement. Educational Studies in Mathematics, Kluwer Academic Publishers, Netherlands, v. 57, n. 1, p. 33-63. July. 2004.

CASTELA, C.; ROMO, A. Des mathématiques à l'automatique: étude des effets de transposition sur la transformée de Laplace dans la formation des ingénieurs. Recherches en Didactique des Mathématiques (RDM), Grenoble, v. 31, n. 1, p. 79-130. Abril. 2011.

CHEVALLARD, Y. L'analyse des pratiques enseignantes en théorie anthropologique du didactique. Recherches en Didactique des Mathématiques (RDM), Grenoble, v. 19, n. 2, p. 221-266. Julio. 1999.

CHEVALLARD, Y. Steps towards a new epistemology in mathematics education. In: BOSCH, M. (Ed.). Proceedings of the 4th Conference of the European Society for Research in Mathematics Education (CERME 4). Barcelona: Universitat Ramon Llull, 2006. p. 21-30.

CHEVALLARD, Y. Enseñar Matemáticas en la Sociedad de Mañana: Alegato a Favor de un Contraparadigma Emergente. REDIMAT - Journal of Research in Mathematics Education, Barcelona, v. 2, n. 2, p. 161 -182. Junio. 2013.

FONSECA, C. Una posible "razón de ser" de la diagonalización de matrices en ciencias económicas y empresariales. In: BRONNER, A. et al. (Ed.). Diffuser les mathématiques (et les autres savoirs) comme outils de connaissance et d'action. Montpellier, Francia: IUFM de l'Académie de Montpellier, 2010. p. 595-614.

GALBRAITH, P. Beyond the low hanging fruit. In: BLUM, W. et al. (Ed.). Modelling and applications in mathematics education: The 14th ICMI study. New York: Springer, 2007. p. 79-88.

GARCÍA, F.J.; GASCÓN, J.; RUIZ-HIGUERAS, L.; BOSCH, M. Mathematical modelling as a tool for the connection of school mathematics, ZDM: The International Journal on Mathematics Education, Springer Berlin Heidelberg, v. 38, n. 3, p. 226-246. June. 2006.

GASCÓN, J. Incidencia del modelo epistemológico de las matemáticas sobre las prácticas docentes. RELIME. Revista latinoamericana de investigación en matemática educativa, Distrito Federal, México, v. 2, n. 4, p. 129-160. Julio. 2001.

GASCÓN, J. Las tres dimensiones fundamentales de un problema didáctico. El caso del algebra elemental. RELIME. Revista Latinoamericana de Investigación en Matemática Educativa, Distrito Federal, México, v. 14, n. 2, p. 203-231. Julio. 2011.

SERRANO, L.; BOSCH, M.; GASCÓN, J. Recorridos de estudio e investigación en la enseñanza universitaria de ciencias económicas y empresariales. UNO: Revista de didáctica de las matemáticas, Barcelona, Núm. 062, p. 39-48. Abril. 2013.

SIERRA, T. Lo matemático en el diseño y análisis de organizaciones didácticas. Los sistemas de numeración y la medida de magnitudes. 2006. 478p. Tesis (Doctoral en Didáctica de las Matemáticas) - Facultad de Educación, Departamento de Didáctica de las Matemáticas, Universidad Complutense de Madrid. 2006.

WINSLØW, C.; BARQUERO, B.; DE VLEESCHOUWER, M.; HARDY, N. An institutional approach to university mathematics education: from dual vector spaces to questioning the world. 
Research in Mathematics Education (RME). Special Issue: University Mathematics Education, Routledge, v. 16, n. 2, p. 95-111. Junio. 2014.

Submetido em Junho de 2014. Aprovado em Fevereiro de 2015. 